\title{
High Precision Agriculture: An Application Of Improved Machine-Learning Algorithms
}

\author{
Jérôme Treboux \\ Institute of Information Systems \\ University of Applied Sciences and Arts Western Switzerland University of Applied Sciences and Arts Western Switzerland \\ Sierre, Switzerland \\ jerome.treboux@hevs.ch
}

\begin{abstract}
This paper presents the performances of machine learning algorithms on aerial images object detection for high precision agriculture. The dataset used focuses on geotagged pictures of vineyards. We demonstrate that advanced machine learning methodologies like Decision Tree Ensemble, outperform state-of-the-art image recognition algorithms generally used within the agriculture field. The innovative approach described here improve object detection and obtain an accuracy of $94.27 \%$ which is an increase of more than $4 \%$ compared to the state-ofthe-art. Finally, methodology and possible developments for high precision agriculture are discussed in this study.

Index Terms-Machine Learning, Decision Tree Ensemble, Random Forests, Prediction, Hyper-spectral images, Agricultural health, Precision agriculture, Image recognition
\end{abstract}

\section{INTRODUCTION}

Nowadays, precision agriculture aims at increasing productivity and maximizing the yields of a crop. The entire crop cycle can benefit from an application of the correct amount of spray (such as water, fertilizers, pesticides or fungicides) at the proper time and place. In parallel, research on multispectral image analysis of agricultural fields is starting to detect diseases in plants [1]. Farmers generate maps of spatial variabilities based on geo-located sensors. These sensors collect many variables and provide historical and realtime information. Recently, Unmanned Aerial Vehicles (UAV) have enabled precision agriculture. These drones are equipped with multispectral or RGB cameras to collect aerial images and create maps of the selected region [2].

High precision agriculture applies mainly to yield monitoring or remote sensing. It is only started to be applied to vineyards on complex landscapes and difficult topographies. To provide better management of vineyards and other cultures, new services are under development around the world. For example, a service for the automatic collection of data related to culture for automatic spotting, designation of zones, and prediction of the evolution of a zone is in preparation [1]. Currently, projects are based on satellites providing highquality images to automatically detect specific zones and generate advanced geographical data [3] [4].

Today, image recognition field massively uses Machine Learning (ML) algorithms, for online images matching or satellite image mapping [5] for example.
The current research is based on the needs above and focuses on the detection of the vine and potential disease on images taken by Unmanned Aerial Vehicles (UAV). UAV are used for multiple reasons such as the price and the accessibility of this type of product, compared to satellites. With an autogenerated flight plan, the drone can fly over a specifically defined zone of the field, carrying products and spraying it out to the designated area.

The present paper will describe how the application of Machine Learning to images taken by a UAV improves the overall performance of automated drone plan generation.

\section{STATE-OF-THE-ART}

As described above, machine learning applies the image recognition broadly and image analysis for research purpose and its use is increasing for business products. Projects are currently underway or have recently been completed to improve the quality of image recognition and object detection. This section reviews and explains these research projects.

\section{A. Pixel-based and Object-oriented classification}

Image processing most basic operation is image classification [6]. Two classification process are mainly used: (1) the pixel-based classification (PBC) and (2) the object-oriented classification (OOC).

The PBC uses conventional statistical techniques. All pixels are categorized into a specific class or thematic. This classification is based on features extracted from the pixel, such as the spectral information and spectral estimated signature.

The OOC classifies objects presents on the picture. Spatialspectral features of the high-resolution (HR) satellite data information is used as the main feature. Last research projects and development for the OOC focuses on the rule-based classifier and the nearest neighbor classifier.

The Pixel-based Classification ignores the impact of mixed pixels by combining the spectral response from the training dataset [7]. In contrast, the $\mathrm{PBC}$ cannot recognize objects bigger than a pixel [8], due to the lack of analysis of the relationship between pixels [9].

By using spectral information like shapes, texture, and relationships between contexts, the OOC increases the object detection accuracy. Furthermore, the detection accuracy is 
improved by the usage of the vector-based GIS and a large set of features like the contextual properties [10] [11] [12].

\section{B. Object-based image analysis by application of nonlinear} scale-space filtering

This research project presents a second powerful and widely used methodology for image recognition. This method analyses images through nonlinear scale-space filtering. Classification of different pictures and various aerial images is provided through a recently developed platform [13].

This framework demonstrates the importance of the qualitative properties and classifier such as multilevel object representation and Support Vector Machine classifier (SVM). Furthermore, it eliminates the need to tune several parameters during the segmentation. These platform algorithms outperform the previous development regarding the final graphical representation and the overall accuracy. Fig. 1 shows the precision and visualization obtain with the explained framework.
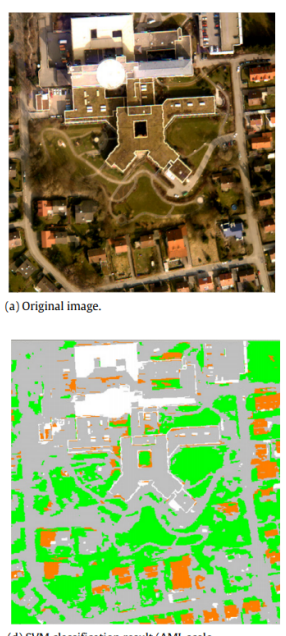

(d) SVM classification result (AML L scale
$100+$ MSEG). Accuracy $=90.16 \%$.

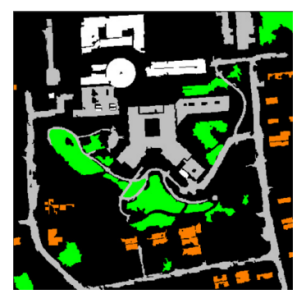

(b) Ground truth data.

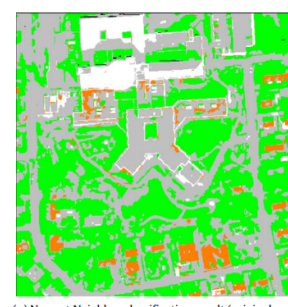

(e) Nearest Neighbor classif
image). Accuracy $=86.72 \%$

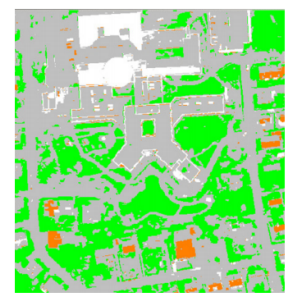
(c) SVM classification result (original
image + MSEG). Accuracy $=89.1 \%$.

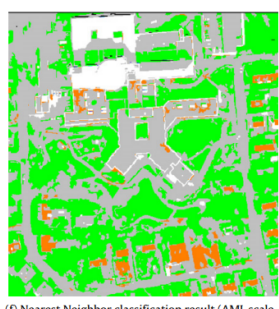

(f) Nearest Neighbor class
$100)$. Accuracy $=87.35 \%$.
Fig. 1. Classification visualization and accuracy including and excluding the AML filtering. The green zones are vegetation, grey zones are the asphalt, the orange zones are the tile roofs and the white zones are the bright roofs.

\section{Vineyard analysis from very High-Resolution Satellite Data}

The research of Karakizi \& al. [4] focuses on the development of automated and efficient agriculture methods. It is presented as a framework and it is based on multispectral data analysis for object classification. Even if multiple research projects are focusing on aerial images analysis for specific zone detection [14] [15] [16] [17] [18], because of the complexity of vineyard fields very few of them address this specific use case. The number of analysis regarding precision agriculture is reduced. This complexity depends on:

- The landscape (vines in the mountains for example)

- The paths and roads going through the vineyards

- Leaves and trunks mixed with metal to maintain the vine

The work presented in [4] describe vine varieties discrimination and applies machine-learning algorithms to reach an overall accuracy close to $92 \%$. The error is generated by multiple factors. The first type of error comes from the ground truth definition which is generated by somehow imprecise human evaluation. The second type of errors is due to a lack of data for some specific classes (certain kind of vines for example) which will be ignored by the algorithms.

\section{DATASET AND DATA PROCESSING}

The dataset is composed of images from five vineyards in the canton of Valais, Switzerland. Many UAV flights generate aerial images and combine GeoTIFF images format to keep the geolocation information. A photogrammetry software produces a full image, with aerial images, containing the overall vineyards to represents the reality of the field to create a flight plan for the drone. These images are produced with software provided by Pix4D [19], a company that develops a suite of photogrammetry software for drone mapping and 3D models.

The application of the research of Lorenzo Comba \& al. [20] generates the baseline based on original UAV images. The baseline is described more in details in the next section.

Because of the Machine Learning methodology used, the dataset is manipulated to match the requirements. The full image of the vineyard is divided into tiles of 30x33 pixels. Because of the size of the tiles, they possibly contain multiple categories: (1) Road, (2) Vineyard and (3) Other (See Fig. 2). The definition of the tile size is based on previous experiments. The tile needs to contain enough data to be detected as an object but precise enough to be classified as a single category. After the pre-processing, the dataset comprises 13'005 images. Each image receives a label containing the category name. When a tile contains at least $30 \%$ of vine, it is categorized as vineyard. This manual process result is summarized in Table I.

TABLE I

DATASET SUMMARY AFTER SUBDIVISION. IT PRESENTS THE CATEGORIES AND NUMBER OF TILES.

\begin{tabular}{|c|c|c|}
\hline Class & Number of tiles & Size of tiles \\
\hline vineyard & 7'022 & 30x33 pixels \\
\hline road & 2 '082 & $30 \times 33$ pixels \\
\hline other & 3'903 & $30 \times 33$ pixels \\
\hline \hline Number of tiles per vineyard & \multicolumn{2}{|c|}{ 2'601 } \\
\hline Total number of tiles & \multicolumn{2}{|c|}{ 13'005 } \\
\hline
\end{tabular}
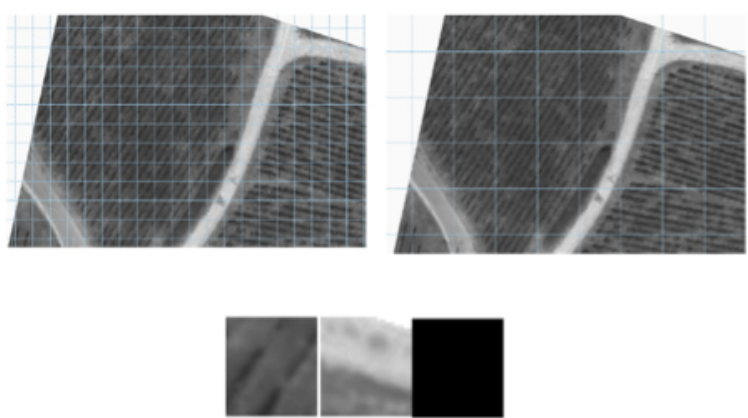

Fig. 2. Vineyard subdivision to obtain 13'005 tiles with a size of 30x33 pixel each. 
Each tile used to train and test the DTE model went through the data preparation process (See Fig. 3). This process is defined as follow:

- Dataset: image opening, labeling with a category the image, features extraction

- Feature elimination: the model generated with the backward features elimination is applied. This removes noisy features

- Prediction: application of the model trained on the training dataset

- Evaluation: scorer generation to diffuse the result and performance of the classification

\section{BASELINE DESCRIPTION}

The baseline is based on the research from Lorenzo Comba $\&$ al. [20], using the dataset described before. This research is mainly based on the image intensity variation and Hough Transform [21] for vine categorization.

First, the dataset quality is controlled. Due to the different color intensities, the object may be differentiated. Fig. 4 shows the original vineyard image with the two selected objects. It demonstrates a visual difference between color intensities.

Fig. 5 confirms the difference in the intensity between the road and the vines. The color intensity is significantly stronger on the vineyard. It reinforces the possibility to use the existing methodology to classify grapes based on color intensity feature.

Morphological processes increase overall accuracy [22]. Two transformations are applied: (1) opening and (2) closing transformations. First, opening transformation removes noise with a succession of erosion and dilation process. Then the closing process applies dilation and erosion to close small missing pixel inside the foreground objects (applied with OpenCV) [23]. These transformations fortify the foreground color and intensity, in this case, the lines of vines. Fig. 6 shows the result of this process with a strong overview of the vineyards. The lines between the vines and the road are white. Some issues because of the small intensity difference with the road on some parts (bottom left of the graph). These parts are wrongly associated with the vineyard.

\section{Vine Detection Improvement With DTE}

The Decision Tree Ensemble (DTE), based on Random Forests, uses features extracted from tiles of the vineyard to create all the necessary trees. The algorithm is based on three groups of features extracted from each tile. It represents 86 features in total, summarized below:

- First order statistics: Min, max, mean, geometric mean, sum, variance, skewness, kurtosis, etc.

- Tamura: Granularity, Contrast, Kurtosis of directionality, Standard Deviation Directionality, Max Directionality and Skewness.

- Haralick: Statistical features based on gray-level cooccurrence matrix.

The feature elimination process is applied to detect and select features that reduce the overall accuracy and increase the computational time. Even if the DTE selects the feature internally with the most information in it, applying backward feature elimination process will help to reduce quicker the number of features and improves the performances. This technique trains a model on $n$ input features. Then, one feature at a time is removed and the model is trained $\mathrm{n}$ times on $\mathrm{n}-1$ input features. The feature producing the smallest increase in the error rate is eliminated. The classification is repeated using n-2 features. An error rate is provided during each iteration. Finally, by selecting the maximum acceptable error rate, the smallest number of features mandatory to train the model is defined [24]. The elimination process determines the minimal amount of features for image classification to 16 features.

Based on an iterative process, fine-tuning of the algorithm is done over similar dataset sampling, revealing a final configuration with the following criteria:

- Split criterion: Information Gain Ratio

- Tree depth: No limitation

- Minimum child node size: No minimum value

- Number of trees: 100

- Rows data sampling method: Stratified

- Attributes data sampling method: Square root

\section{RESUlTs AND Discussion}

This section presents the results of the baseline algorithms application and the solution using Decision Tree Ensemble. The overall accuracy and the standard error are proposed for each methodology.

The baseline and the Decision Tree Ensemble are applied to specific vineyards, with different landscape features (size, orientation, slope). Results are presented in Table II. Accuracy variation rise between vineyards, but results are not significantly different based on the standard error.

TABLE II

PERFORMANCES COMPARISON BETWEEN THE BASELINE AND THE DECISION TREE ENSEMBLE ON TWO DIFFERENT TYPE OF VINEYARDS.

\begin{tabular}{|l|c|c|c|c|}
\hline & \multicolumn{2}{|c|}{ Vineyard 1 } & \multicolumn{2}{c|}{ Vineyard 2 } \\
& Baseline & DTE & Baseline & DTE \\
\hline Acc & $90.02 \%$ & $94.27 \%$ & $89.60 \%$ & $94.32 \%$ \\
\hline Std err & $\pm 1.17 \%$ & $\pm 1.13 \%$ & $\pm 1.01 \%$ & $\pm 1.14 \%$ \\
\hline
\end{tabular}

Decision Tree Ensemble model generates an accuracy at the Equal Error Rate of $94.275 \% \pm 1.14 \%$ (See Table III). Vines are detected correctly with some errors due to mixed categories on specific tiles. This mix comes from a wrong manual labeling; multiple classes are present on the same tile or there are missing data within a particular category. Furthermore, DTE is very stable throughout all iterations executed and keeps a stable accuracy.

In comparison, the baseline generates an accuracy at the Equal Error Rate of 90.076\% $\pm 1.09 \%$ (See Table IV).

Experiments are significantly different with a better performance using the DTE. The number of misclassifications with the baseline is higher. Table III and IV show the classification results for each method. The vineyard tiles are correctly 


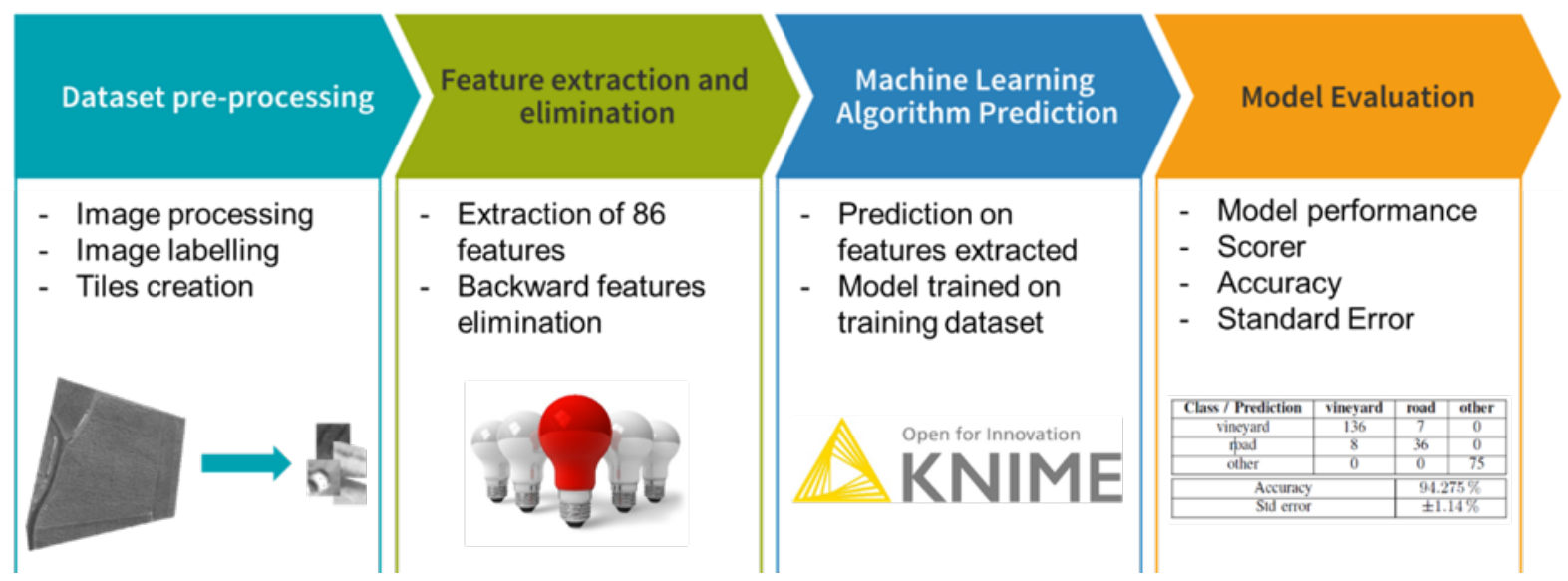

Fig. 3. Overview of the DTE model generation. This extract of the DTE structure shows the sequence of decision for a tile classification.

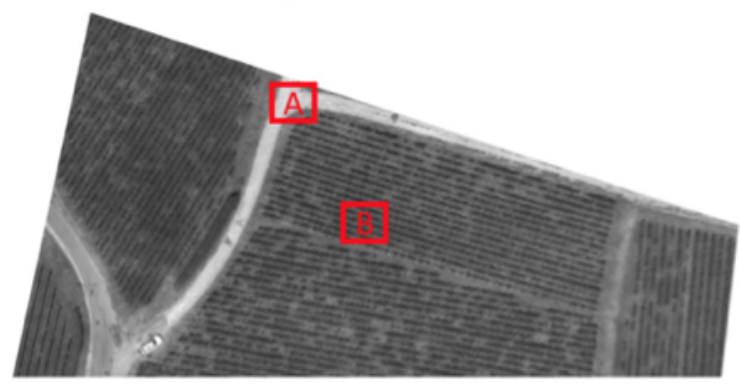

Fig. 4. Original GeoTIFF vineyard image. (A) is a sample of a road and (B) a sample of a vine for intensity feature analysis.

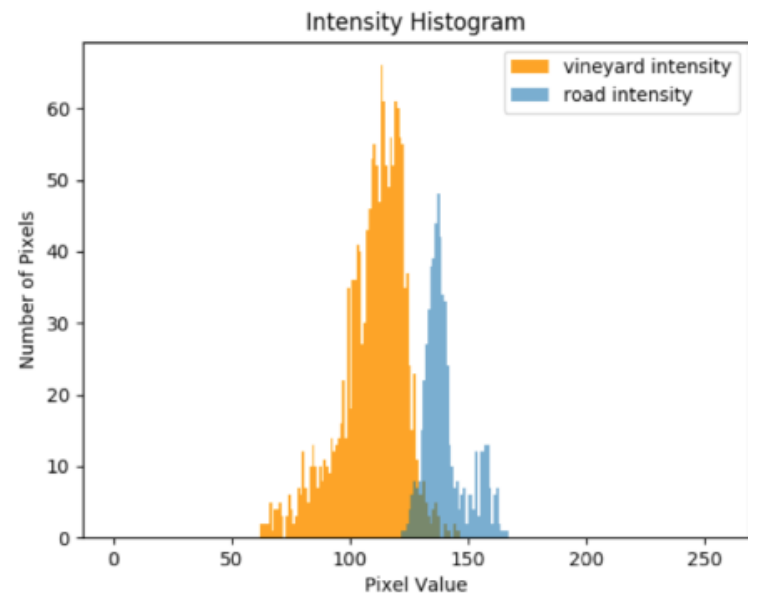

Fig. 5. Graphical representation of the color intensity for a road and for a vine. It shows the number of pixel per intensity level for each category.

classified at $95.1 \%$ (136 over 143 tiles) with the DTE and only at $86.7 \%$ (124 over 143 tiles) with the baseline methodology. In opposite, the roads are better detected with the baseline $(86.3 \%)$ than with the DTE $(81.8 \%)$. The other objects detection is stable with both algorithms.
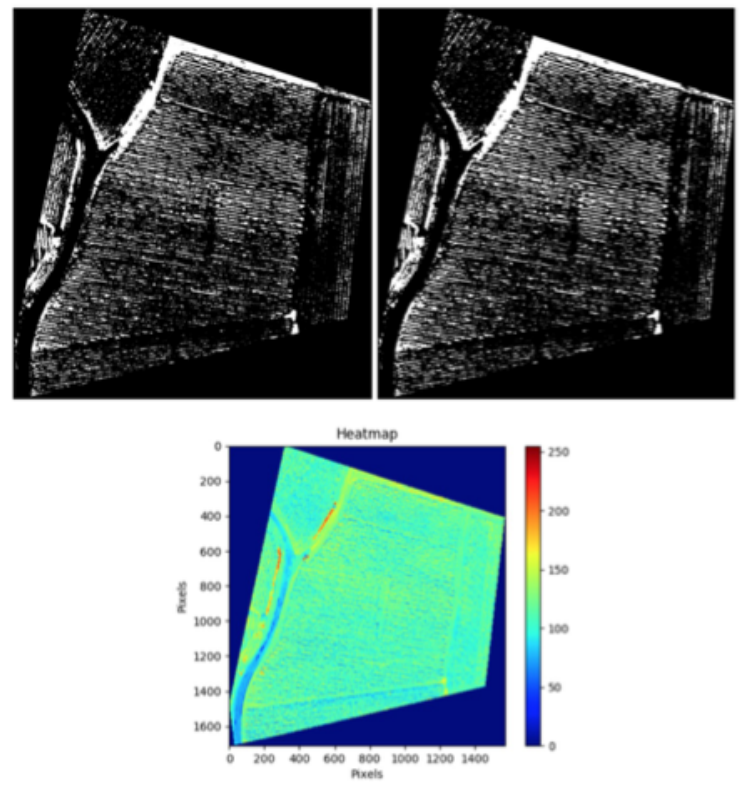

Fig. 6. Results after an intensity augmentation and a reduction of the noise with morphological processes. The top left image shows the transformed image, the top right image is the original black and white image. The heat map (bottom) shows the color intensity with line of vine in blue.

\section{CONCLUSION}

Detecting objects in a vineyard using the DTE produces the best accuracy in comparison with the baseline. Furthermore, the number of objects of type vineyard misclassification is smaller than with the baseline. Misclassification of vineyard objects have an impact on the diffusion of the treatment products. Parts of the vineyard will not be managed. In opposite, spraying a small part of the road has a smaller impact on the precision agriculture efficiency.

This high accuracy vine detection is used as a preliminary project for automated flight plan generation. As presented in this study, the accuracy is increased drastically by the implementation of Machine Learning algorithms. It becomes 
interesting for the High Precision Agriculture to use this process and help farmers within their field management. Furthermore, the DTE is a simple algorithm for a computer to apply with a reduced number of features, obtained with the backward feature elimination process (See Fig. 7).

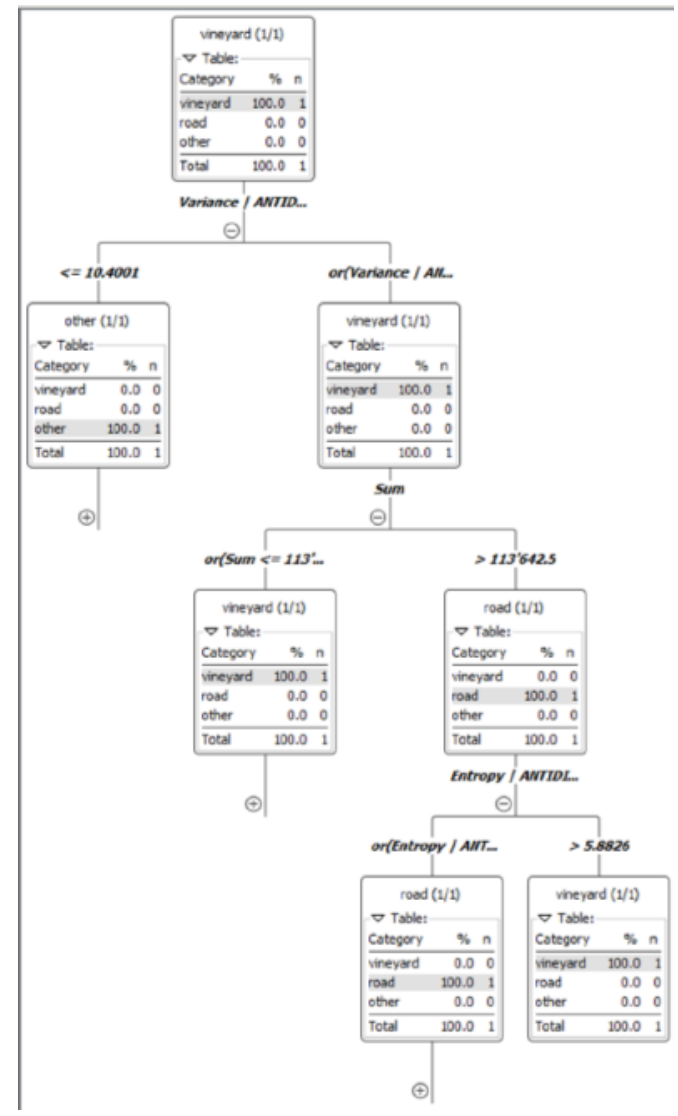

Fig. 7. Overview of the DTE model generation. This extract of the DTE structure shows the sequence of decision for a tile classification.

TABLE III

Overall Confusion Matrix FOR THE DTE MODEL ON THE FULl TEST DATASET (262 SAMPLES)

\begin{tabular}{|l|c|c|c|}
\hline Class / Prediction & vineyard & road & other \\
\hline vineyard & 136 & 7 & 0 \\
\hline road & 8 & 36 & 0 \\
\hline other & 0 & 0 & 75 \\
\hline \hline Accuracy & \multicolumn{3}{|c|}{$94.275 \%$} \\
\hline Std error & \multicolumn{3}{|c|}{ $\pm 1.14 \%$} \\
\hline
\end{tabular}

TABLE IV

OVERALl CONFusion MATRIX FOR THE BASELINE ON THE FULL DATASET (262 SAMPLES).

\begin{tabular}{|l|c|c|c|}
\hline Class / Prediction & vineyard & road & other \\
\hline vineyard & 124 & 19 & 0 \\
\hline road & 6 & 38 & 0 \\
\hline other & 0 & 1 & 74 \\
\hline \hline Accuracy & \multicolumn{3}{|c|}{$90.076 \%$} \\
\hline Std error & \multicolumn{3}{|c|}{ $\pm 1.09 \%$} \\
\hline
\end{tabular}

\section{ACKNOWLEDGMENT}

This research was supported by the Institute of Information Systems of the University of Applied Sciences and Arts Western Switzerland (HES-SO).

\section{REFERENCES}

[1] J. Treboux, D. Genoud and R. Ingold, "Decision Tree Ensemble Vs. NN Deep Learning: Efficiency Comparison For A Small Image Dataset," 2018 International Workshop on Big Data and Information Security (IWBIS). Jakarta, IEEE, 2018.

[2] G. Popkin, "Satellite alerts track deforestation in real time," nature.com, 530(7591), 392-393, 2016.

[3] C. Anderson, "Agricultural Drones Relatively cheap drones with advanced sensors and imaging capabilities are giving farmers new ways to increase yields and reduce crop damage," MIT Technology Review, May/June 2014.

[4] C. Karakizi, M. Oikonomou, and K. Karantzalos, "Vineyard Detection and Vine Variety Discrimination from Very High Resolution Satellite Data," Remote Sens, 8, 235, 2016.

[5] B. Schultz, M. Formaggio, A. R. Del' Arco Sanchez, L. Barreto Luiz and C.N Atzberger, "Self-Guided Segmentation and Classification of MultiTemporal Landsat 8 Images for Crop Type Mapping in Southeastern Brazil,' Remote-Sensing, 14482-14508, 2015.

[6] S. D. Jawak, P. Devliyal and A. J. Luis, "A Comprehensive Review on Pixel Oriented and Object Oriented Methods for Information Extraction from Remotely Sensed Images with a Special Emphasis on Cryospheric Applications," ScriRes, 4, 177-195, 2015.

[7] D. Lu and Q. Weng, "Spectral Mixture Analysis of the Urban Landscape in Indianapolis with Landsat ETM+ Imagery," Photogrammetric Engineering and Remote Sensing, 70, 1053-1062, 2004.

[8] P. Corcoran, A. Winstanley and P. Mooney, "Segmentation Performance Evaluation for Object Based Remotely Sensed Image Analysis," International Journal of Remote Sensing, 31, 617-645, 2010.

[9] H. Sridharan and F. Qiu, "Developing an Object Based Hyperspectral Image Classifier with a Case Study Using WorldView-2 Data," Photogrammetric Engineering and Remote Sensing, 79, 1027-1036, 2013.

[10] Z. Chen, X. Ning and J. Zhang, "Urban Land Cover Classification Based on WorldView-2 Image Data," IEEE International Symposium on Geometrics for Integrated Water Resource Management. Lanzhou, 2012.

[11] P. Aplin and G. Smith, "Advances in Object Based Image Classification," The International Archives of Photogrammetry, Remote Sensing and Spacial Information Sciences, 37, 725-728, 2008.

[12] D. Liu and F. Xia, "Assessing Object.Based Classification: Advantages and Limitations," Remote Sensing Letters, 1, 187-194, 2010.

[13] A. Tzotsos, K. Karantzalos and D. Argialas, "Object-based image analysis through nonlinear scale-space filtering," Journal of Photogrammetry and Remote Sensing, 66, 2-16, 2011.

[14] S. Rapinel, B. Clément, S. Magnanon, V. Sellin and L. Hubert-Moy, "Identification and mapping of natural vegetation on a coastal site using WorldView-2 satellite image," J. Environ. Manag., 144, 236-246, 2014.

[15] R. Lottering and O. Mutanga, "Optimizing the spatial resolution of WorldView-2 imagery for discriminating forest vegetation at subspecies level in KwaZulu-Natal," Geocarto Int. South Africa, 2015.

[16] M. Immitzer, C. Atzberger and T. Koukal, "Tree Species Classification with Random Forest Using Very High Spatial Resolution 8-ban WorldView-2 Satellite Data," Remote Sens., 4, 2661-2693, 2012.

[17] R. Pu and S. Landry, "A comparative analysis of high spatial resolution IKONOS and WorldView-2 imagery for mapping urban tree species," Remote Sens., 124, 516-533, 2012.

[18] O. Mutango, E. Adam and M. Cho, "High density biomass estimation for wetland vegetation using WorlView-2 imagery and random forest regression algorithm," Int. J. Appl. Earth Obs. Geoinform., 18, 399406, 2012.

[19] B. Draeyer, and C. Strecha, "White paper: How accurate are UAV surveying methods?," 2014.

[20] L. Comba, P. Gay, J. Primicerio and D. R. Aimonino, "Vineyard detection from unmanned aerial systems images," Computers and Electronics in Agriculture, 114, 78-87, 2015.

[21] D.H. Ballard, "Generalizing the Hough transform to detect arbitrary shapes," Pattern Recognition, Volume 13, Issue 2, 111-122, 1981. 
[22] M.L. Comer, E.J. Delp, "Morphological operations for color image processing," J. Electron. Imag. 8(3), 1 July 1999.

[23] J. Treboux, and D. Genoud, "Improved Machine Learning Methodology for High Precision Agriculture," 2018 Global Internet of Things Summit (GIoTS). Bilbao, Spain, pp. 1-6, IEEE, June 2018.

[24] KNIME.org, "Seven Techniques for Data Dimensionality Reduction," KNIME.org Blog, May 2015. 